\title{
Solution of Singular Integral Equations of the First Kind with Cauchy Kernel
}

\author{
Subhabrata Mondal ${ }^{1 *}$, B.N. Mandal $\left.\right|^{2}$
}

\begin{abstract}
In this paper an analytic method is developed for solving Cauchy type singular integral equations of the first kind, over a finite interval. Chebyshev polynomials of the first kind, $T_{n}(x)$, second kind, $U_{n}(x)$, third kind, $V_{n}(x)$, and fourth kind, $W_{n}(x)$, corresponding to respective weight functions $W^{(1)}(x)=\frac{1}{\sqrt{1-x^{2}}}, W^{(2)}(x)=\sqrt{1-x^{2}}, W^{(3)}(x)=$ $\sqrt{\frac{1+x}{1-x}}$, and $W^{(3)}(x)=\sqrt{\frac{1-x}{1+x}}$, have been used to obtain the complete analytical solutions for four different cases. Keywords: Singular integral equation, Cauchy Kernel, Chebyshev polynomials, Weight function
\end{abstract} 2010 AMS: Primary 45E05

${ }^{1}$ Department of Applied Mathematics, University of Calcutta, 92,A.P.C. Road, Kolkata-700009, India

2 Physics and Applied Mathematics Unit, Indian Statistical Institute, 203, B.T Road, Kolkata-700108, India

*Corresponding author: subhamath644@gmail.com

Received: 20 August 2018, Accepted: 22 January 2019, Available online: 22 March 2019

\section{Introduction}

Consider the Cauchy type singular integral equations (CSIEs) of the form

$$
\int_{-1}^{1} \frac{\phi(t)}{(t-x)} d t=f(x), \quad-1<x<1
$$

where $f(x)$ is given real valued function and $\phi(t)$ is unknown function to be determined. Integral equation of the form (1.1) occurs in a variety of mixed boundary value problems of mathematical physics (cf. [1], [2], [3]).

Kim [4] solved CSIEs by using Gaussian quadrature and choose the zeros of Chebyshev polynomials of first and second kinds as the collocation and abscissa points. Chakrabarti and Berge [5] have proposed an approximate method for solving CSIEs using polynomial approximation of degree $n$ and collocation points chosen to be the zeros of the Chebyshev polynomial of the first kind for all cases. Mandal and Panja [6] obtained the numerical solution of the second kind CSIEs using Daubechies scale function. Abdulkawi [7] uses differential transform method for the numerical solution of CSIEs of the first kind. Mondal and Mandal [8] used Chebyshev polynomials of first and second kind to obtain the exact solution of a simple hypersingular integral equation.

In this paper, we present the analytical solution of CSIEs (1.1). There are four basically important and interesting cases of equation (1.1), as given by the following :

Case I. $\Phi(x)$ is unbounded at both the end-point $x= \pm 1$. 
Case II. $\Phi(x)$ is unbounded at the end $x=-1$, but bounded at the end $x=+1$.

Case III. $\Phi(x)$ is bounded at the end $x=-1$, but unbounded at the end $x=+1$.

Case IV. $\Phi(x)$ is bounded at both the end-point $x= \pm 1$.

It is well known that the complete analytical solutions of equation (1.1) for the above four cases, are given by the following expressions:

Case I :

$$
\phi(x)=\frac{A_{0}}{\sqrt{1-x^{2}}}-\frac{1}{\pi^{2} \sqrt{1-x^{2}}} \int_{-1}^{1} \frac{\sqrt{1-t^{2}} f(t)}{t-x} d t
$$

where $A_{0}$ is an arbitrary constant,

\section{Case II :}

$$
\phi(x)=-\frac{1}{\pi^{2}} \sqrt{\frac{1-x}{1+x}} \int_{-1}^{1} \sqrt{\frac{1+t}{1-t}} \frac{f(t)}{t-x} d t
$$

\section{Case III :}

$$
\phi(x)=-\frac{1}{\pi^{2}} \sqrt{\frac{1+x}{1-x}} \int_{-1}^{1} \sqrt{\frac{1-t}{1+t}} \frac{f(t)}{t-x} d t,
$$

Case IV : In this case the solution exists iff $\int_{-1}^{1} \frac{f(t)}{\sqrt{1-t^{2}}} d t=0$ and the solution is given by

$$
\phi(x)=-\frac{\sqrt{1-x^{2}}}{\pi^{2}} \int_{-1}^{1} \frac{f(t)}{\sqrt{1-t^{2}}(t-x)} d t .
$$

\section{Chebyshev polynomials and their integral transforms}

The Chebyshev polynomials of the first kind $T_{n}(x)$, second kind $U_{n}(x)$, third kind $V_{n}(x)$, and fourth kind $W_{n}(x), n=0,1,2, \ldots \ldots$ are defined (cf. [9]) as follows :

$$
\begin{aligned}
& T_{n}(x)=\cos n \theta, \quad U_{n}(x)=\frac{\sin (n+1) \theta}{\sin \theta}, \\
& V_{n}(x)=\frac{\cos \left(n+\frac{1}{2}\right) \theta}{\cos \frac{1}{2} \theta}, \quad W_{n}(x)=\frac{\sin \left(n+\frac{1}{2}\right) \theta}{\sin \frac{1}{2} \theta},
\end{aligned}
$$

where $x=\cos \theta, 0 \leq \theta \leq \pi$.

It is well known that the Chebyshev polynomials of first and second kinds are integral transforms of each other with respect to weighted Hilbert kernels, as given by

$$
\int_{-1}^{1} \frac{T_{n}(t)}{\sqrt{1-t^{2}}(t-x)} d t=\pi U_{n-1}(x),-1<x<1
$$

and

$$
\int_{-1}^{1} \frac{\sqrt{1-t^{2}} U_{n}(t)}{(t-x)} d t=-\pi T_{n+1}(x),-1<x<1
$$


Here the integral is to be interpreted as a Cauchy principal value integral.

It is further known that the third and fourth kind polynomials are similarly related :

$$
\int_{-1}^{1} \sqrt{\frac{1+t}{1-t}} \frac{V_{n}(t)}{(t-x)} d t=\pi W_{n}(x),-1<x<1
$$

and

$$
\int_{-1}^{1} \sqrt{\frac{1-t}{1+t}} \frac{W_{n}(t)}{(t-x)} d t=\pi V_{n}(x),-1<x<1 .
$$

Further, we will also use the result

$$
\int_{-1}^{1} \frac{T_{n}(t)}{\sqrt{1-t^{2}}} d t= \begin{cases}\pi & , n=0 \\ 0 & \text { otherwise }\end{cases}
$$

\section{Method of the solution}

\section{Case I.}

To obtain the solution of the integral equation (1.1) satisfying the end conditions given in Case I, we assume an expansion of $\phi(x) \quad(-1 \leq x \leq 1)$ in terms of Chebyshev polynomials of first kind $T_{n}(x)$ as

$$
\phi(x)=\frac{1}{\sqrt{1-x^{2}}} \sum_{n=0}^{\infty} a_{n} T_{n}(x),-1<x<1
$$

where $a_{n}(n=0,1,2, \ldots)$ are unknown constants. Substituting (3.1) in the left side of (1.1) and assuming the validity of interchange of the order of integration and summation we obtain

$$
\sum_{n=1}^{\infty} a_{n}\left[\pi U_{n-1}(t)\right]=f(t),-1<t<1
$$

wherein the results

$$
\int_{-1}^{1} \frac{T_{0}(t)}{\sqrt{1-t^{2}}(t-x)} d t=0
$$

and (2.1) have been utilized.

Multiplying both sides of equation (3.2) by $\frac{\sqrt{1-t^{2}}}{t-x}$ and then integrating with respect to $t$ from -1 to 1 we obtain

$$
\pi \sum_{n=1}^{\infty} a_{n} \int_{-1}^{1} \frac{\sqrt{1-t^{2}} U_{n-1}(t)}{(t-x)} d t=\int_{-1}^{1} \frac{\sqrt{1-t^{2}} f(t)}{(t-x)} d t,-1<t<1
$$

Now using the result (2.2) in (3.3), we obtain

$$
\sum_{n=0}^{\infty} a_{n} T_{n}(x)=a_{0}-\frac{1}{\pi^{2}} \int_{-1}^{1} \frac{\sqrt{1-t^{2}} f(t)}{(t-x)} d t,-1<t<1 .
$$


Using equation (3.4) in equation (3.1), we obtain the solution of the integral equation (1.1) as given by (1.2).

\section{Case II.}

In this case $\phi(x)$ can be written in terms of Chebyshev polynomials of fourth kind $W_{n}(x)$ as

$$
\phi(x)=\sqrt{\frac{1-x}{1+x}} \sum_{n=0}^{\infty} b_{n} W_{n}(x),-1<x<1
$$

where $b_{n}(n=0,1,2, \ldots)$ are unknown constants.

In a similar manner we obtain

$$
\sum_{n=0}^{\infty} b_{n}\left[-\pi V_{n}(t)\right]=f(t),-1<t<1
$$

wherein the result (2.4) has been utilized.

Multiplying both sides of equation (3.6) by $\sqrt{\frac{1+t}{1-t}} \frac{1}{t-x}$ and then integrating with respect to $t$ from -1 to 1 we obtain

$$
-\pi \sum_{n=0}^{\infty} b_{n} \int_{-1}^{1} \sqrt{\frac{1+t}{1-t}} \frac{V_{n}(t)}{t-x} d t=\int_{-1}^{1} \sqrt{\frac{1+t}{1-t}} \frac{f(t)}{t-x} d t,-1<t<1
$$

Now, using the result (2.3) in equation (3.7), we obtain

$$
\sum_{n=0}^{\infty} b_{n} W_{n}(x)=-\frac{1}{\pi^{2}} \int_{-1}^{1} \sqrt{\frac{1+t}{1-t}} \frac{f(t)}{t-x} d t,-1<t<1 .
$$

Using equation (3.8) in equation (3.5), we obtain the solution of the integral equation (1.1) as given by (1.3).

\section{Case III.}

Here $\phi(x)$ can be written in terms of Chebyshev polynomials of third kind $V_{n}(x)$ as

$$
\phi(x)=\sqrt{\frac{1+x}{1-x}} \sum_{n=0}^{\infty} c_{n} V_{n}(x),-1<x<1
$$

where $c_{n}(n=0,1,2, \ldots)$ are unknown constants.

In a similar way we obtain

$$
\sum_{n=0}^{\infty} c_{n}\left[\pi W_{n}(t)\right]=f(t),-1<t<1
$$

wherein the result (2.3) has been used.

Multiplying both sides of equation (3.10) by $\sqrt{\frac{1-t}{1+t}} \frac{1}{t-x}$ and then integrating with respect to $t$ from -1 to 1 we obtain

$$
\pi \sum_{n=0}^{\infty} c_{n} \int_{-1}^{1} \sqrt{\frac{1-t}{1+t}} \frac{W_{n}(t)}{t-x} d t=\int_{-1}^{1} \sqrt{\frac{1-t}{1+t}} \frac{f(t)}{t-x} d t,-1<t<1
$$

Now, using the result (2.4) in equation (3.11), we obtain

$$
\sum_{n=0}^{\infty} c_{n} V_{n}(x)=-\frac{1}{\pi^{2}} \int_{-1}^{1} \sqrt{\frac{1-t}{1+t}} \frac{f(t)}{t-x} d t,-1<t<1 .
$$


Using equation (3.12) in equation (3.9), we obtain the solution of the integral equation (1.1) as given by (1.4).

\section{Case IV.}

To obtain the bounded solution of the integral equation (1.1), we can expand $\phi(x)(-1 \leq x \leq 1)$ in terms of Chebyshev polynomials of second kind $U_{n}(x)$ as

$$
\phi(x)=\sqrt{1-x^{2}} \sum_{n=0}^{\infty} d_{n} U_{n}(x),-1<x<1
$$

where $d_{n}(n=0,1,2, \ldots)$ are unknown coefficients.

Proceeding in the same manner we obtain

$$
\sum_{n=0}^{\infty} d_{n}\left[-\pi T_{n+1}(t)\right]=f(t)
$$

wherein the result (2.2) has been utilized.

By using the result (2.5) in (3.14) we obtain

$$
\int_{-1}^{1} \frac{f(t)}{\sqrt{1-t^{2}}} d t=0
$$

which is the solvability criterion for the existence of the bounded solution.

Multiplying both sides of equation (3.14) by $\frac{1}{\sqrt{1-t^{2}}(t-x)}$ and then integrating with respect to $t$ from -1 to 1 we obtain

$$
-\pi \sum_{n=0}^{\infty} d_{n} \int_{-1}^{1} \frac{T_{n+1}(t)}{\sqrt{1-t^{2}}(t-x)} d t=\int_{-1}^{1} \frac{\sqrt{1-t^{2}} f(t)}{\sqrt{1-t^{2}}(t-x)} d t,-1<t<1
$$

Now using the result (2.1) in equation (3.15), we obtain

$$
\sum_{n=0}^{\infty} d_{n} U_{n}(x)=-\frac{1}{\pi^{2}} \int_{-1}^{1} \frac{f(t)}{\sqrt{1-t^{2}}(t-x)} d t,-1<t<1 .
$$

Using equation (3.16) in equation (3.13), we obtain the solution of the integral equation (1.1) as given by (1.5).

\section{Illustrative examples}

Consider the singular integral equation

$$
\int_{-1}^{1} \frac{\phi(t)}{(t-x)} d t=x^{4}+5 x^{3}+2 x^{2}+x-\left(\frac{11}{8}\right), \quad-1<x<1
$$

with the exact solutions for different cases as given by (cf. [10])

Case (I) : $\phi(t)=\frac{1}{\pi \sqrt{1-t^{2}}}\left[t^{5}+5 t^{4}+\frac{3}{2}\left(t^{3}-t^{2}\right)-\frac{5}{2} t-\frac{9}{8}\right]$.

Case (II) : $\phi(t)=-\frac{1}{\pi} \sqrt{\frac{1-t}{1+t}}\left[t^{4}+6 t^{3}+\frac{15}{2} t^{2}+6 t+\frac{7}{2}\right]$.

Case (III) : $\phi(t)=\frac{1}{\pi} \sqrt{\frac{1+t}{1-t}}\left[t^{4}+4 t^{3}-\frac{5}{2} t^{2}+t-\frac{7}{2}\right]$.

Case (IV) : $\phi(t)=-\frac{1}{\pi} \sqrt{1-t^{2}}\left[t^{3}+5 t^{2}+\frac{5}{2} t+\frac{7}{2}\right]$. 
Here $f(x)=x^{4}+5 x^{3}+2 x^{2}+x-\left(\frac{11}{8}\right)$. For the solution of the integral equation (4.1) satisfying the end conditions given in Case I, we can write $\phi(x)$ in the form given by (3.1). In a similar manner, we can obtain the expression given by (3.4).

Using Eq. (3.4) in Eq. (3.1) and then using the result

$$
\int_{-1}^{1} \frac{\sqrt{1-t^{2}} t^{k}}{(t-x)} d t=-\pi x^{k+1}+\sum_{i=0}^{k-1} \frac{1+(-1)^{i}}{4} \frac{\Gamma\left(\frac{1}{2}\right) \Gamma\left(\frac{i+1}{2}\right)}{\Gamma\left(\frac{i+4}{2}\right)} x^{k-i-1}, j=1,2, . .
$$

we obtain the solution of the IE (4.1) given in Case I.

In a similar way we can obtain the solutions of the integral equation (4.1) for all the other cases by using the methods described separately.

Remark 4.1. The method of solution for obtaining the solution of (1.1) for the Case I is well known in the literature but is given here for the sake of completeness. The methods adopted for other cases, although simple, appear to be not known, and that is why these are given here.

Acknowledgement The first author thanks CSIR for the award of a research fellowship (CSIR award letter no09/028(0971)/2016-EMR-I, Dated: 19/10/2016).

\section{References}

[1] N. I. Mushkelishvili, Singular Integral Equations, Noordhoff, Groningen, 1953.

[2] F.D. Gakhov, Boundary Value Problems, Addison-Wesley, 1966.

[3] P. A. Martin, F. S. Rizzo, On boundary integral equations for crack problems, Proc. R. Soc. Lond. Ser. A Math. Phys. Eng. Sci., 421 (1989), 341-345.

[4] S. Kim, Solving singular integral equations using Gaussian quadrature and overdetermined system, Appl. Math. Comput., 35 (1998), 63-71.

[5] A. Chakrabarti, V. G. Berghe, Approximate solution of singular integral equations, Appl. Math. Lett., 17 (2004), 553-559.

[6] M. M. Panja, B. N. Mandal, Solution of second kind integral equation with Cauchy type kernel using Daubechies scale function, J. Comput. Appl. Math., 241 (2013), 130-142.

[7] M. Abdulkawi, Solution of Cauchy type singular integral equations of first kind by using differential transform method, Appl. Math. Model., 39 (2015), 2107-2118.

[8] S. Mondal, B. N. Mandal, A note on the solution of a simple hypersingular integral equation, Glob. J. Pure Appl. Math., 13 (2017), 1959-1964.

[9] J.C. Mason, Chebyshev polynomials of the second, third and fourth kinds in approximation, indefinite integration, and integral transforms, J. Comput. Appl. Math., 49 (1993), 169-178.

[10] Z. K. Eshkuvatov, N. M. A. Nik Long, M. Abdulkawi, Approximate solution of singular integral equations of the first kind with Cauchy kernel, Appl. Math. Lett., 22 (2009), 651-657. 